\title{
Improving patient access at a movement disorder clinic by participating in a Process Improvement Program
}

\author{
Alan Goodridge, Douglas Woodhouse, Janet Barbour
}

\begin{abstract}
Our multi-disciplinary neurology team were dissatisfied with long access times for consultation for new referrals. We participated in a rapid process improvement workshop and a structured improvement process. Over a six-month period we were able to reduce our access time for initial appointment for patients with suspected movement disorders from 133 to 20 days. We implemented a 'carousel' multi-disciplinary appointment and a standardised clinic form that improved the flow of patients and that we estimate will save 150 hours of physician time and 320 hours of administrative time per year.
\end{abstract}

\section{Problem}

Our specialist team provides tertiary care services at an academic health centre for a large geographical region. Access times for consultation at a multi-disciplinary clinic for neurological patients were very long, which was a concern for both family physicians and our neurology team.

Because of our long wait list, it was necessary to triage referrals by perceived urgency and secretarial staff often had to 'squeeze' urgent patients into full schedules, causing clinics to run over-time.

\section{Background}

Access times are an issue in most publicly funded health systems. In Canada, wait time targets have been defined for a number of referral reasons. However in 2012, after several years of 'slight progress' wait times in many provinces increased (1).

Wait times for the ambulatory care clinic at our nearest comparable hospital were 8 days for urgent patients, 39 days for semi-urgent and 115 days for non-urgent patients (2). Anecdotal reports from another Canadian province indicate wait times for neurologist consultation of between six months and a year (3). This suggests that long wait times to access neurological consultation is a problem in other Canadian jurisdictions.

Rapid process improvement workshops have been used in industries outside of healthcare for many years to identify problems and plan improvements. Recently, hospitals have adapted tools such as Lean and utilised process improvement workshops with impressive results, demonstrating that these techniques also have applicability in healthcare $(4,5)$.

\section{Baseline measurement}

Aggregate appointment data from the previous year was available for the multi-disciplinary clinic from hospital data systems. This provided us with a baseline for the ratio of new versus follow-up, Parkinson versus non-Parkinson and the attendance rate at the clinic.

We felt that additional data would be required to evaluate our performance. We therefore kept a clinic log, which provided insight into clinic performance without creating excessive administrative overhead. On the daily agenda, physicians documented actual time worked (start and stop times, as well as any additional time required for related administration such as documentation).

During a two day process improvement workshop our team mapped clinical processes, identified process flow bottlenecks and planned improvements. Improvement theories including Lean and Theory of Constraints were applied and we identified many issues causing inefficiency and frustration (See supplementary files: Table: Issues Identified).

See supplementary file: ds1921.pdf - "Table: Issues Identified"

\section{Design}

We designated an improvement team consisting of representatives from all roles that are involved with our clinical service. This included physicians, nurses, secretaries, managers and physiotherapists. An explicit commitment of support for the process improvement project was obtained from senior management. Apart from the services of an external facilitator and a 2-day release from clinical activities, no additional resources were provided to the team. During the process improvement workshop we utilised a prioritization matrix to define four major improvement themes.

First, it was felt that a focus on movement disorders at the multidisciplinary clinic (rather than seeing patients with a range of diagnoses) would reduce access times, increase efficiency and help us to provide optimal patient care. Identifying 'probable movement disorder' referrals as early as possible would allow us to book 
patients directly into the movement disorder clinic. The majority of these patients have Parkinson's Disease, although other conditions such as Huntington's disease and dystonia are included.

Second, a central intake for new referrals would allow us to balance new patients between all physicians working at the clinic, resulting in a fairer distribution of workload and shorter average access times.

Third, we wanted patients to see all members of the multidisciplinary team during every appointment. Flow between multidisciplinary providers was previously ad-hoc, patients were seen by the first available team member and did not always see all members of the multi-disciplinary team (nurse, physiotherapist and neurologist). This resulted in duplication of activity, such as patients repeating their clinical history and physical exam multiple times during their appointment.

Fourth, we felt that quality of care would improve if we could increase the frequency of follow-up appointments and improve our accessibility for new referrals and unstable patients. It was clear that this could only be achieved by ensuring that follow-up appointments for relatively stable patients did not consume an everincreasing proportion of our available time.

\section{Strategy}

Specific interventions were implemented to address the 4 highest priority issues. For each, an implementation plan was developed defining the implementation activities, a deadline, a responsible person and the expected benefit (which we defined as either time savings or higher quality).

Implementation began immediately after the process improvement workshop. Within several weeks we implemented a multidisciplinary assessment form and a 'carousel' appointment. Patients were seen initially by a nurse, then by a physiotherapist and finally by a neurologist. Each provider's assessment was documented on the same form. We provided a standardized, comprehensive assessment and eliminated the duplication associated with our previous, ad-hoc process. Reduced variation in workflow enabled us to balance appointment durations for each provider, resulting in improved patient flow.

Previously the physician dictated a consultation letter to the family physician, which was transcribed and mailed. Using our new form, we were able to immediately fax the assessment and plan to the family physician and eliminate dictations.

All patients now see every member of the multi-disciplinary team at every appointment. We feel that this helps us to provide optimal patient care. At the same time we have experienced a reduction in perceived work "pressure" and a reduction in overtime clinics.

We have also implemented a centralized referral for new movement disorder patients. Previously, each physician's secretary would independently book patients. A single secretary is now responsible for scheduling the movement disorder clinic. Using a list of referral key words, secretaries review all incoming referrals to identify referrals for 'probable movement disorder' and immediately book these patients at the movement disorder clinic. New patients are assigned to the first available neurologist, rather than the neurologist to whom the referral was sent. Family physicians retain the option to refer to an individual neurologist with the understanding that this may result in a longer access time.

Previously, no blocked appointment slots were available for new patients or for patients that needed to be seen urgently, these patients were squeezed into the agenda when necessary. We initially blocked appointment slots that came free (due to cancellations or other reasons) for urgent or new patients, and concurrently blocked slots in the future schedule. This has significantly reduced our access time and reduced urgent fit-ins. Additional capacity has been created by extending follow-up intervals and by discharging stable patients from general clinics.

A myriad of additional improvements have contributed to improved performance. We identified more than 23 potential improvements during the initial workshop, many of which were relatively simple and have already been implemented. We did not attempt to define the specific contribution of each improvement but expected that the cumulative impact would positively impact our performance metrics.

Finally, we plan to hold process improvement workshops on a quarterly basis to identify new improvement ideas, prioritise changes and plan implementation as part of a continuous improvement strategy.

\section{Results}

Our results reflect the initial six months of the project (as of 25 March 2013). We have achieved an impressive reduction in access time for patients, as measured by the third next available appointment. Access time (measured as the third next available appointment) is now 20 days compared to 133 days at the beginning of the project.

The percentage of patients seen at the clinic with Parkinson disease has risen to $88 \%$ from a baseline of $27 \%$. New patients now account for $23 \%$ of appointments from $8.4 \%$. Attendance has risen to an average of $93 \%$ from $81 \%$.

We estimate that the carousel appointment and multi-disciplinary clinic form will save 150 hours of physician time and 320 hours of administrative time per year by eliminating duplication and reducing dictations. The number of patients seen per hour declined slightly after implementing carousel appointments, in line with our expectations. We have increased the frequency of follow-up appointments for movement disorder patients.

Despite a decrease in access times, our wait list for new appointments has not dropped. Our rate of referrals is greater than our rate of discharge, and if this continues we will eventually run out of capacity to see new and urgent patients. Extending follow-up intervals for stable patients in our general neurology clinics has partially mitigated the impact of this imbalance. However we 
continue to face a capacity challenge.

See supplementary file: ds1920.pdf - "Figures of Results"

\section{Lessons and limitations}

We identified several key success factors for the implementation of sustainable clinical improvements. We believe that most of these are generalizable to clinical process improvement at other sites and for other patient groups.

1. Process improvement projects should be the responsibility of the multi-disciplinary clinical team. Our improvement team consisted of representatives from all roles that are involved with our clinical service. This included physicians, nurses, secretaries, managers and physiotherapists. Each team member shared their unique perspectives on work processes and issues, which ensured a deeper, shared understanding of our work. By engaging all members of the team, we were confident of reaching broad consensus leading to alignment on issues and improvement plans.

2. We focused on improvements that our clinical team could implement without the approval, support or involvement of external stakeholders. This allowed us to quickly implement changes once we had reached consensus within our team. Senior management support was obtained, but apart from the services of an external facilitator and a two day release from clinical activities no additional resources were required.

3. We chose a three month deadline to present our initial results to an external audience. This deadline mandated that we focus on practical, attainable improvements and contributed to a continued focus on implementation activities. We continued to implement improvements following the initial three month period with on-going improvement in performance metrics. We plan to hold quarterly process improvement workshops to review results, identify new issues and plan new improvements. Developing a structure to support continuous improvement has contributed to the sustainability of changes and on-going improvement in key performance indicators.

\section{Conclusion}

Our multi-disciplinary neurology team participated in a 2-day rapid process improvement workshop followed by a structured improvement process over a 6-month period. During this time we were able to reduce our access time for initial appointment for patients with suspected movement disorder from 133 to 20 days. We implemented a 'carousel' multi-disciplinary appointment and a standardized clinic form that improved the flow of patients and that we estimate will save 150 hours of physician time and 320 hours of administrative time per year.

\section{References}

1. Shedding Light on Canadian's Total Wait for Care: Report Card on Wait Times in Canada. Wait Time Alliance. June
2012.

(http://www.waittimealliance.ca/media/2012reportcard/WTA 2012-reportcard_e.pdf) [Accessed 26 May 2013]

2. Neurology Wait Times: Nova Scotia Wait Time Information. Province of Nova Scotia. (http://waittimes.novascotia.ca/procedure/neurology) Accessed 14 April 2013

3. Neurologists Want of Service Shortage in Ontario. CBC. (http://www.cbc.ca/news/health/story/2008/02/27/toneurologists.html) [Accessed 26 May 2013]

4. Bohmer R, Ferlins E. Virginia Mason Medical Center. Harvard Business School. 2008.

5. Ford A, Williams J, Spencer M, et.al. Reducing door-toneedle times using Toyota's lean manufacturing principles and value stream analysis. Stroke. 2012 Dec;43(12):3395-8. PMID: 23138440

\section{Declaration of interests}

Dr. Douglas Woodhouse was funded by Newfoundland and Labrador Medical Association to provide facilitation services during the process improvement program.

\section{Acknowledgements}

Robert Ritter, Dr. Oscar Howell, Janet Templeton, Leona Lewis, Elizabeth Kennedy, Dr. Kyna Squarey, Denise Murphy, Jennifer Shears, Ruth Wade, Dr. Mark Stefanelli, Janet Brown, Brenda Peddle 